\title{
Las cualidades arquitectónicas de las aulas y su relación con la ideación creativa ${ }^{1}$.
}

\section{Architectural qualities of classrooms and their relation with creative ideation.}

\section{Cómo citar:}

Elizondo, A., De la Fuente, L.A., \& Mireles, A. (2018). Las cualidades arquitectónicas de las aulas y su relación con la ideación creativa. Designia 6(1), 9-27

${ }^{1}$ Estudio que formó parte de la investigacion de tesis de Maestría titulada Desarrollo de estrategias para el logro de ambientes creativos en las escuelas de diseño, realizada en la Facultad de Arquitectura de la Universidad Autonoma de Nuevo León (México).

*Arquitecta con Maestría en Ciencias con Orientación en Gestión e Innovación del Diseño por la Universidad Autónoma de Nuevo León, UANL, México. Actualmente es profesora en la Facultad de Arquitectura de dicha universidad en Monterrey, México. arq.andreaes@gmail.com, andrea.elizondosl@uanl.edu.mx ORCID: 0000-0001-5702-7595

**Arquitecto con Doctorado en Comunicación Visual en Arquitectura y Diseño (Universitat Politècnica de Catalunya BarcelonaTech, Barcelona). Actualmente es Profesor Investigador en la Facultad de Arquitectura de la Universidad Autónoma de Nuevo León, UANL, México, y se encuentra investigando la experiencia de los edificios mediante el uso del equipo de eye-tracking o rastreo ocular. alfonso.dlfuentesrz@uanl.edu.mx luisalfonsodelafuente@hotmail.com ORCID: 0000-0003-3937-0503

\footnotetext{
***Arquitecto con Maestría en Urbanismo por la Universidad Nacional Autónoma de México, con Doctorado en Filosofía con Orientación en Arquitectura y Asuntos Urbanos por la Universidad Autónoma de Nuevo León, UANL, México. Actualmente es profesor en la Facultad de Arquitectura de la UANL, México adrianbmireles@gmail.com ORCID: 0000-0002-0020-6848
}

\section{Palabras clave:}

Creatividad, aulas, arquitectura, ambientes creativos.

\section{Key words:}

Creativity, classrooms,

architecture, creative environments.

Recibido: 12/06/2018

Aceptado: 17/10/2018 
Resumen:

Si bien las personas pueden ser creativas en mayor o menor medida, el potencial creativo está condicionado por el entorno. Por lo tanto, es esencial proveer ambientes adecuados para propiciar el pensamiento creativo. La investigación empírica de la cual se deriva este artículo buscó detectar las características funcionales y ambientales requeridas para facilitar el proceso de ideación en alumnos de Arquitectura. En desarrollo del estudio, estudiantes de la Facultad de Arquitectura de la Universidad Autónoma de Nuevo León (México) evaluaron diferentes aulas en términos de sus cualidades arquitectónicas y el grado de facilidad con que se verifican en su interior tareas relacionadas con el estudio de la disciplina y la generación de ideas creativas. Los resultados obtenidos muestran correlaciones entre la capacidad para propiciar la creatividad y condiciones como la altura, el color, el sonido, la iluminación y la temperatura.

\section{Abstract}

People can be creative to a greater or lesser extent, nevertheless, this creative potential is conditioned by the environment. Therefore, it is essential to provide adequate environments in order to encourage the development of creative thinking. The empiric study this article stem from aimed to identify functional and environmental qualities that are required to facilitate the creative process in Architecture students. In researching, students from the Universidad Autónoma de Nuevo León's Faculty of Architecture (Mexico) assessed various classrooms taking into account the degree of ease or difficulty to carry out activities related to such discipline and generate creative ideas there. The results show correlations between the capability of fostering creativity and conditions such as height, color, sound, lighting and temperature. 
Uno de los principales retos para las escuelas de diseño consiste en generar ambientes que estimulen el proceso creativo de los estudiantes. Ante esto, el objetivo de la investigación aquí presentada fue detectar en las aulas las cualidades favorecedoras de dicho proceso. De acuerdo con Vigovsky (2011) el ambiente siempre influye de forma determinante en las personas. Este puede propiciar un mejor desempeño en la realización de actividades relacionadas con la producción creativa, por lo tanto, en algunos casos estimula la creatividad, si bien en otros quizá la bloquee o la inhiba. Para Csikszentmihalyi (1998), lo más importante en relación con la creatividad es el medio ambiente, donde subyacen las características arquitectónicas de los espacios. Entre las cualidades de estos se encuentran el color, la altura, la temperatura, la acústica y la iluminación.

En cuanto a esta última, Kahn (2015) señala que la luz artificial nunca podrá igualar los matices creados por la del día. Esa incidencia de la luz natural sobre la materia puede aprovecharse al intervenir, pues en Arquitectura, cuando dicha iluminación se maneja conscientemente, hace "vibrar" el espacio y "transporta" a las personas a estados de ánimo trascendentes. Además, el cuerpo humano está diseñado para vivir en el día con la iluminación del sol y por la noche con la oscuridad (Steidle \& Werth, 2013). Según Arango-Díaz (2016), en ambientes escolares y laborales, la luz blanca solar trae beneficios psicológicos, fisiológicos y económicos. Por ende, es la mejor para el desempeño de los alumnos.

En relación con los colores, Kivi (2017) menciona que tener varios de estos en un aula inspira la creatividad, promueve un mejor desempeño en los estudiantes y puede ayudarles a solucionar problemas más fácilmente. En un estudio realizado por Rui (2009), se analizó cuál color podría servir para estimular la creatividad. Seis diferentes pruebas de imágenes en una computadora con fondos rojo, azul y blanco fueron resueltas por 600 participantes. Quienes se expusieron al fondo azul superaron el test de creatividad, en tanto las personas expuestas al rojo obtuvieron mejores resultados en memoria y atención.

En lo concerniente a la altura, esta permite lograr mejor ventilación, iluminación y sensación de amplitud en un edificio, lo cual conlleva al bienestar psíquico del usuario. Sobre este particular, Lindwell (2011) denomina "efecto catedral" al fenómeno de la influencia en la mente humana de la altura de los espacios. Aquellos con techos más elevados llevan a la abstracción, libertad y creatividad, mientras que donde estos son bajos la atención se centra en cosas concretas. 
Respecto al sonido, Mehta (2012) señala que la extrema tranquilidad dentro de un lugar es benéfica para la concentración durante la lectura o la revisión de números pero inhibe el pensamiento creativo, pues al intensificar el enfoque se impide pensar abstractamente. En contraste, el exceso de sonidos perjudica la creatividad al reducir la atención. Por consiguiente, es necesario un punto medio. Un poco de ruido promueve un "procesamiento disfluente", esto es, falta de fluidez mental que activa la cognición abstracta, lo cual, a su vez, mejora la creatividad. Para Mehta, esta se impulsa mediante una cantidad ideal de 70 decibeles, por tal razón, un lugar de $50 \mathrm{db}$ sería muy silencioso y uno de $85 \mathrm{db}$ demasiado ruidoso y molesto. Ambos, entonces, causarían desconcentración.

La temperatura ambiental es también importante. De acuerdo con Seppanen (2005), la temperatura óptima para ofrecer una sensación de comodidad a las personas y propiciar la creatividad es de $25^{\circ} \mathrm{C}$. Por su parte, Hedge (2004) indica que la productividad al trabajar varía según las condiciones asociadas con este factor. Sobre tal punto, un aula no debería ser menor a $20^{\circ} \mathrm{C}$ ni mayor a $26^{\circ} \mathrm{C}$.

\section{Metodología}

En la primera etapa del estudio se realizó un sondeo con los estudiantes de la Facultad de Arquitectura (FARQ) de la Universidad Autónoma de Nuevo León (UANL), inscritos en el periodo agosto-diciembre de 2016, quienes cursaban de primero a décimo semestre. Se les preguntó cuáles aulas dentro de la Facultad, en las que se impartían asignaturas prácticas, se consideraban las mejores y las peores para tener ideas creativas. Según los resultados, las tres aulas con un potencial alto para la creatividad son: 1114 (en adelante llamada A1, figura 4), 1443 (A2, figura 6) y 4215 (A3, figura 8). Por su parte, las tres con bajo potencial para tal fin son: 2312 (B1, figura 10), 1331 (B2, figura 12) y 4212 (B3, figura 14). En la tabla 1 se presentan las especificaciones generales de todas las aulas. 


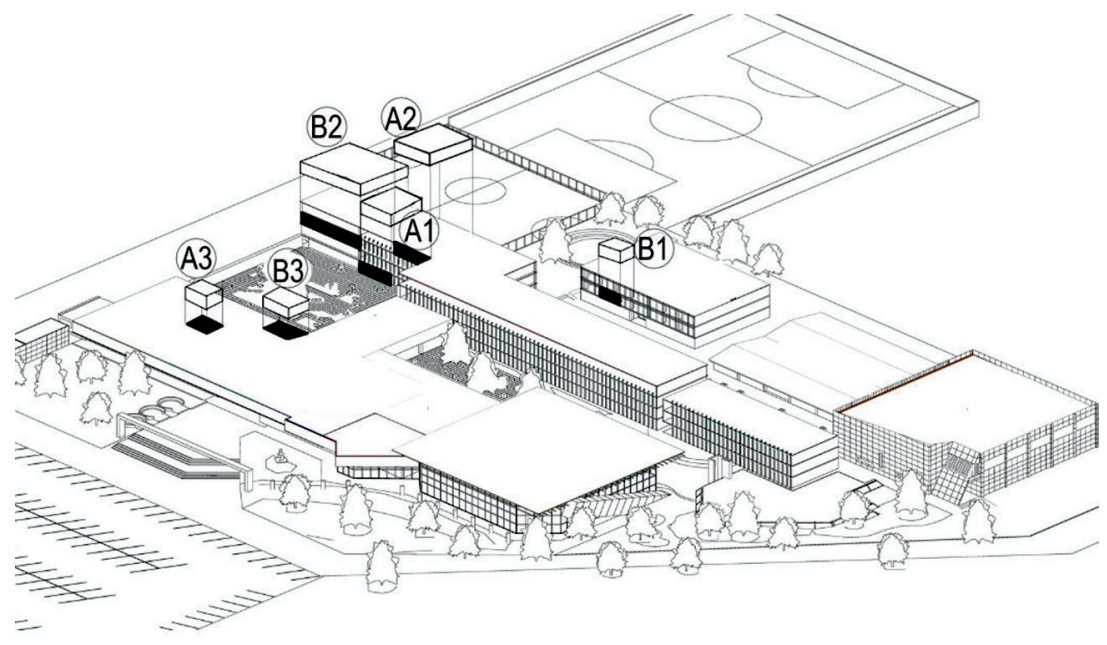

Figura 1. Isometría de la FARQ, UANL. Fuente: autores a partir de la imagen elaborada por el Departamento de Logistica e Imagen de la FARQ, UANL, disponible en http://arquitectura.uanl.mx/instalaciones

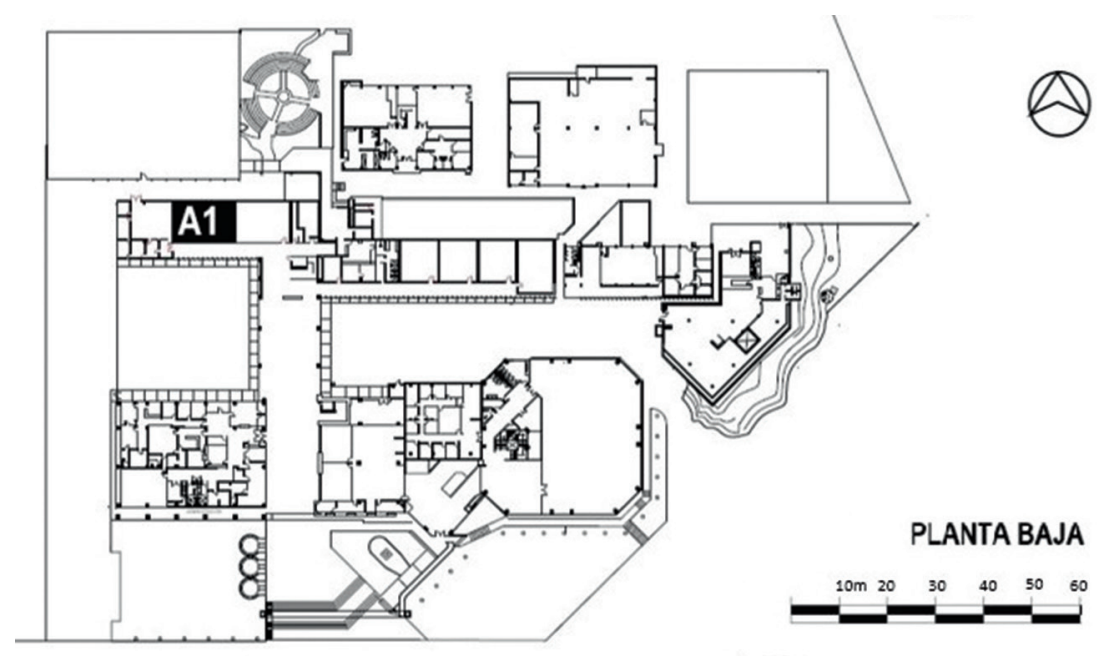

Figura 2. Planta baja de la FARQ, UANL. Fuente: autores. 

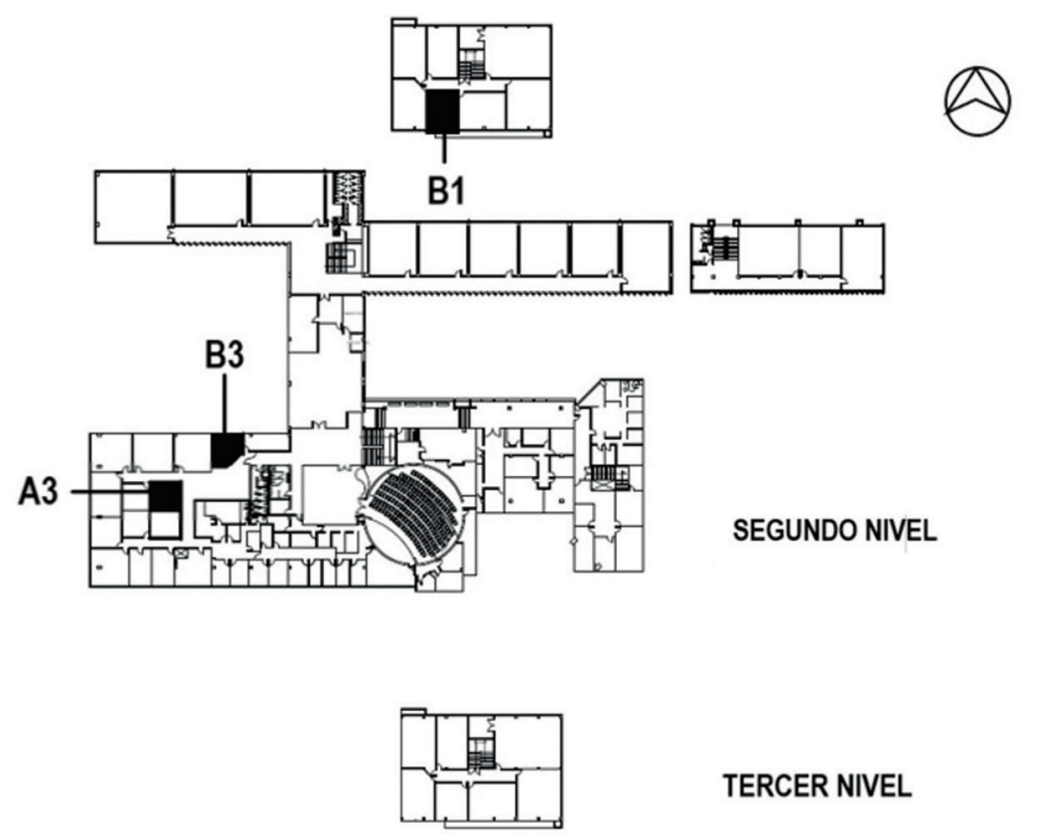

TERCER NIVEL
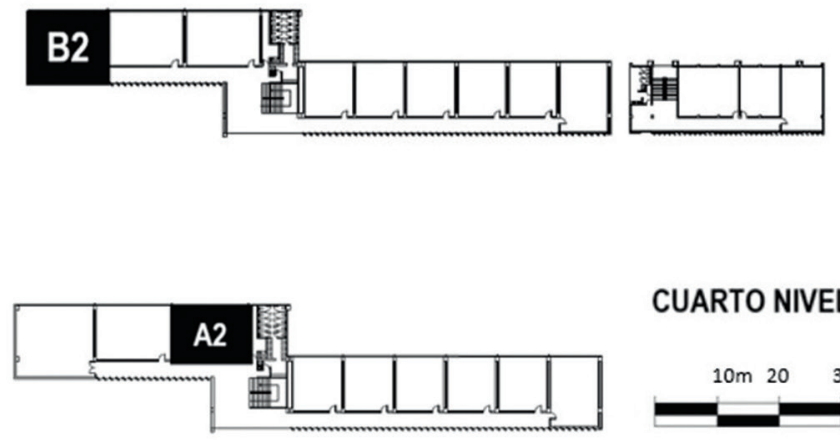

CUARTO NIVEL

Figura 3. Segundo, tercer y cuarto nivel de la FARQ, UANL.

En una segunda etapa, tras conocer las aulas consideradas como de alto y bajo potencial para la creatividad, se aplicó una encuesta sobre cada una a grupos de estudiantes. De las 20 preguntas formuladas, 15 se referían a las cualidades de los espacios y 5 indagaban por las actividades allí realizadas. Los grupos estuvieron conformados por entre 10 y 15 alumnos, quienes calificaron de 0 a 100 cada aspecto mostrado. En todos los casos, el cuestionario fue respondido a las 12 del día. Se analizó la confiabilidad (Hernández, Fernández \& Baptista, 2014) de la encuesta con un estudio de consistencia interna Alfa de Cronbach y con la correlación de Pearson. Esta última se utilizó para generar una matriz de correlación y explicar las relaciones de cada variable. 
La A1 fue la primera aula analizada mediante la aplicación de la encuesta a 14 estudiantes de cuarto semestre. Ubicada en la planta baja del edificio principal de la FARQ, su característica más notoria es ser escalonada. Posee una isóptica que permite una buena visibilidad desde cualquier punto, sus dimensiones son $8.80 \mathrm{~m}$ de ancho por $10.20 \mathrm{~m}$ de largo y su altura es variable, pues debido a su diseño disminuye de $2.70 \mathrm{~m}$ a 2.10 en la parte posterior. El material interior es ladrillo cerámico, pintado de gris claro. Cuenta con dos grandes ventanales en los extremos izquierdo y derecho, cuya altura de antepecho varía. Su iluminación natural es adecuada y se refuerza con luz cenital artificial gracias a lámparas de vapor de mercurio bajo el cielo de plafón de yeso, de color blanco. Como en sus cercanías se sitúa un área verde, es común observar estudiantes en el exterior.

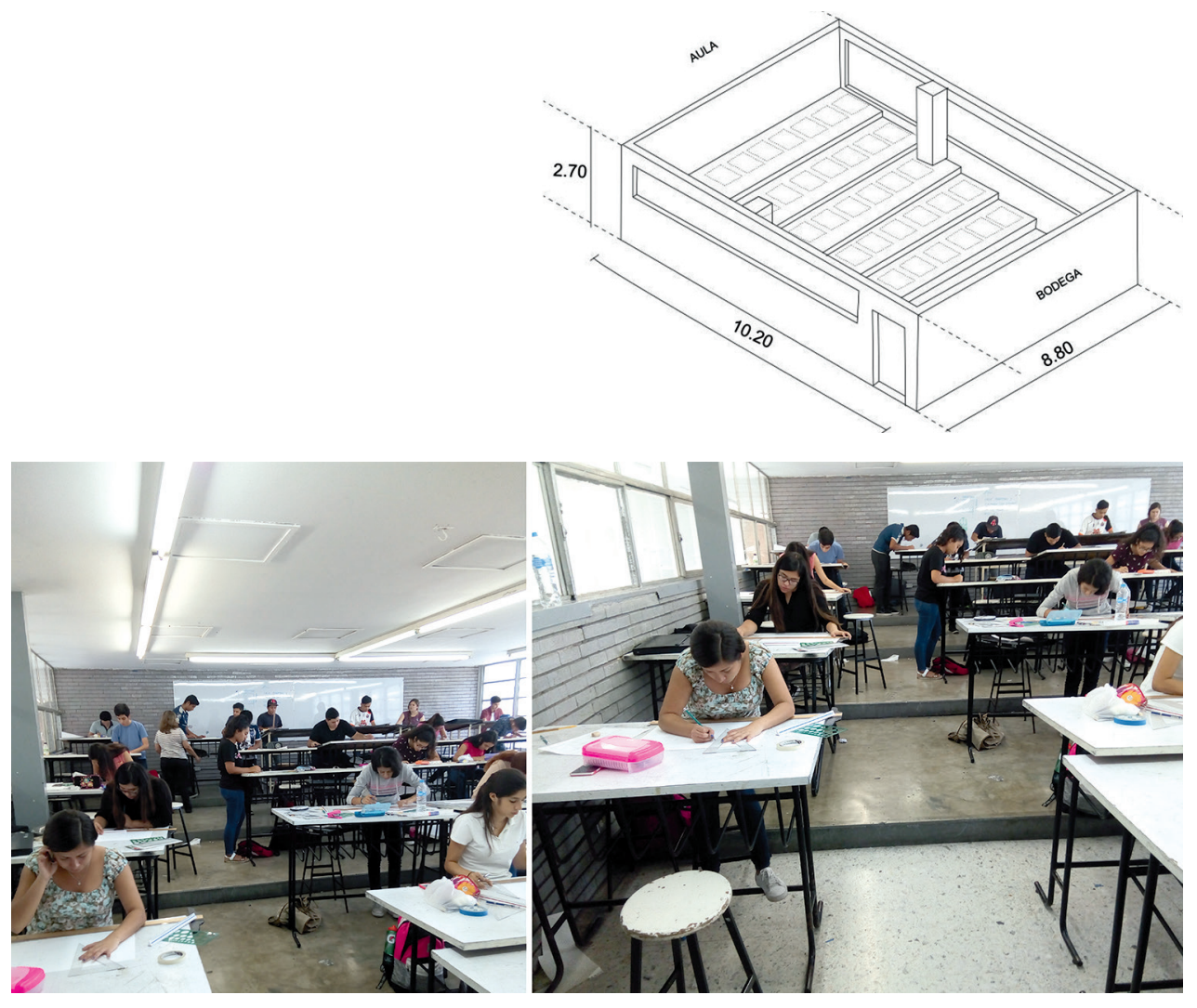

Figura 4. Isometría del aula A1 de la FARQ, UANL.

Figura 5. Fotos del aula A1 de la FARQ, UANL.

Fuente: autores. 


\begin{tabular}{|c|c|c|c|c|c|c|c|}
\hline & Aula & $\mathbf{A 1}$ & A2 & A3 & B1 & B2 & B3 \\
\hline \multirow{3}{*}{ Mobiliario } & Restiradores & 36 & 42 & $\mathrm{n} / \mathrm{a}$ & 12 & 64 & $\mathrm{n} / \mathrm{a}$ \\
\hline & Sillas & 65 & 50 & 15 & 12 & 64 & 15 \\
\hline & Mesas & $\mathrm{n} / \mathrm{a}$ & $\mathrm{n} / \mathrm{a}$ & 2 & $\mathrm{n} / \mathrm{a}$ & $\mathrm{n} / \mathrm{a}$ & 2 \\
\hline \multirow{3}{*}{ Veterinaria } & Porcentaje de ventanas en superficie envolvente & $40 \%$ & $40 \%$ & $50 \%$ & $20 \%$ & $20 \%$ & $70 \%$ \\
\hline & Antepechos & & & & & & \\
\hline & Luz natural & & & & & & \\
\hline \multirow{3}{*}{ Iluminación } & Paneles de iluminación led & & & & & & \\
\hline & Tubos fluorescentes & & & & & & \\
\hline & Lámparas herméticas led & & & & & & \\
\hline \multirow{3}{*}{$\begin{array}{l}\text { Acabados } \\
\text { en muros }\end{array}$} & Ladrillo & & & & & & \\
\hline & Liso & & & & & & \\
\hline & Panel de vidrio & & & & & & \\
\hline \multirow{3}{*}{ Piso } & Concreto & & & & & & \\
\hline & Cerámico & & & & & & \\
\hline & Diferentes niveles & & & & & & \\
\hline \multirow{3}{*}{ Estructura } & Vigas aparentes & & & & & & \\
\hline & Columnas aparentes & & & & & & \\
\hline & Sin estructura aparente & & & & & & \\
\hline \multirow{2}{*}{ Puertas } & Puerta tipo bandera & & & & & & \\
\hline & Puerta doble & & & & & & \\
\hline
\end{tabular}

Para el aula A2 se encuestaron 11 estudiantes de quinto semestre. Esta se ubica en el cuarto nivel, realizado como una ampliación vertical en una etapa posterior al edificio original. Al igual que las demás del nivel, sus dimensiones son $6 \mathrm{~m}$ de ancho por $10 \mathrm{~m}$ de fondo y $3.50 \mathrm{~m}$ de altura. No cuenta con diseño isóptico y sus materiales interiores son tableros de yeso (tablarroca) pintados de gris oscuro. Tiene dos grandes ventanales en los extremos, con antepechos a $1 \mathrm{~m}$ de altura y cerramiento a $3 \mathrm{~m}$, los cuales brindan una vista panorámica de la ciudad universitaria. Gracias a esta amplia área vidriada, la iluminación natural es muy buena y a esto se suma la luz artificial cenital de lámparas de vapor de mercurio. Un punto negativo es su estructura metálica expuesta que inhibe la acústica. 

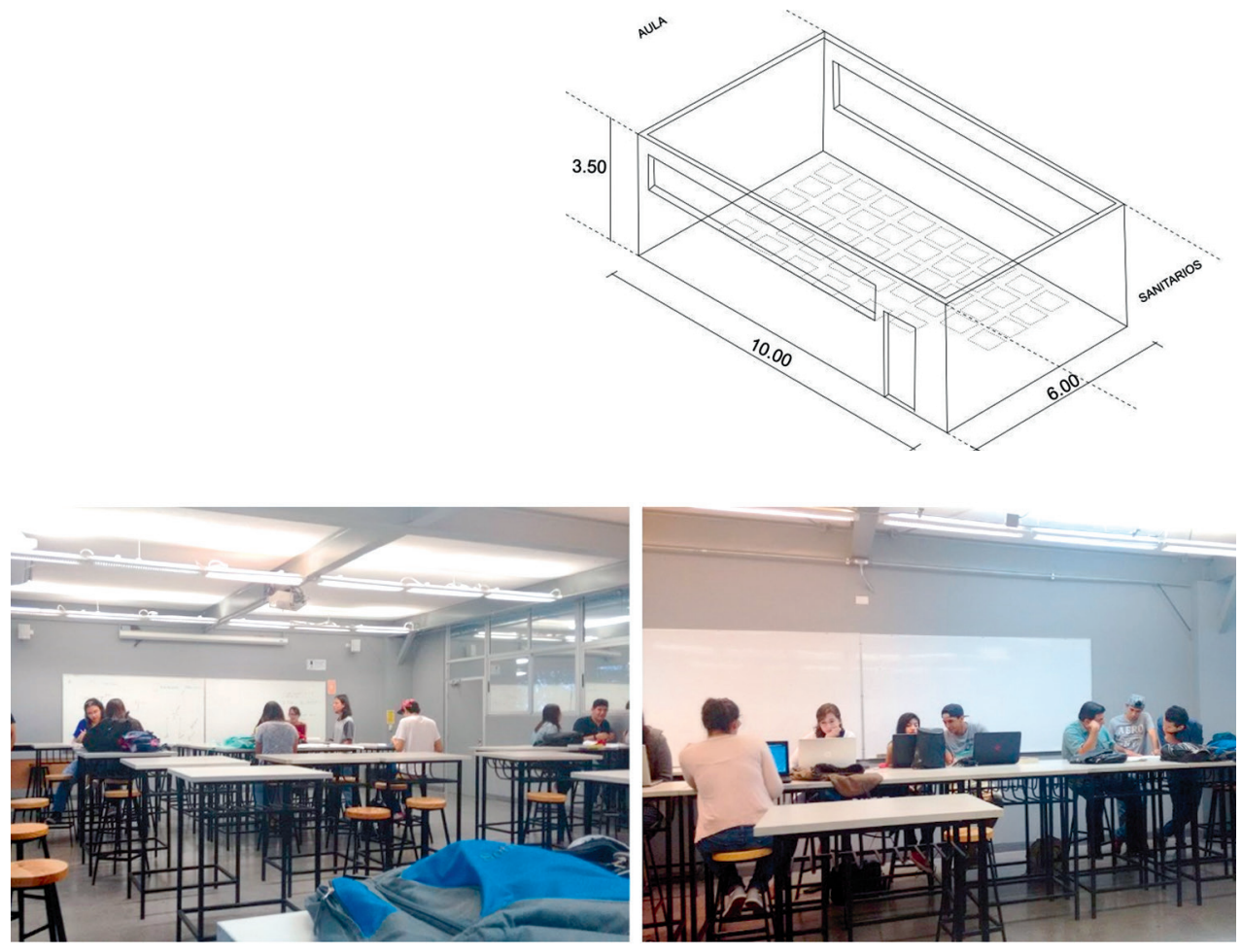

Figura 6. Isometría del aula A2 de la FARQ, UANL.

Figura 7. Fotos del aula A2 de la FARQ, UANL.

Fuente: autores.

La encuesta para el aula A3 se aplicó a 13 estudiantes de noveno semestre. Localizada en el área de posgrado de la Facultad, en la intersección de dos pasillos, está expuesta a una constante circulación de personas. Se le emplea para impartir clases de licenciatura por las mañanas, y en las tardes, asignaturas de posgrado. Se le puede considerar pequeña, pues sus medidas son de $4 \mathrm{~m}$ por lado y $2.50 \mathrm{~m}$ de altura al nivel de plafón. Dos de sus cuatro muros contiguos son de vidrio de piso a 
techo, lo que permite ver el exterior (recepción y sala de posgrado). Sus materiales interiores son tableros de yeso de color beige y cancelería de aluminio con vidrio claro. Carece de ventilación e iluminación natural, por lo tanto, lo primera se logra mediante un sistema de aire acondicionado que regula la temperatura interna, mientras que la luz proviene de lámparas de vapor de mercurio intercaladas en el plafón de paneles de yeso.
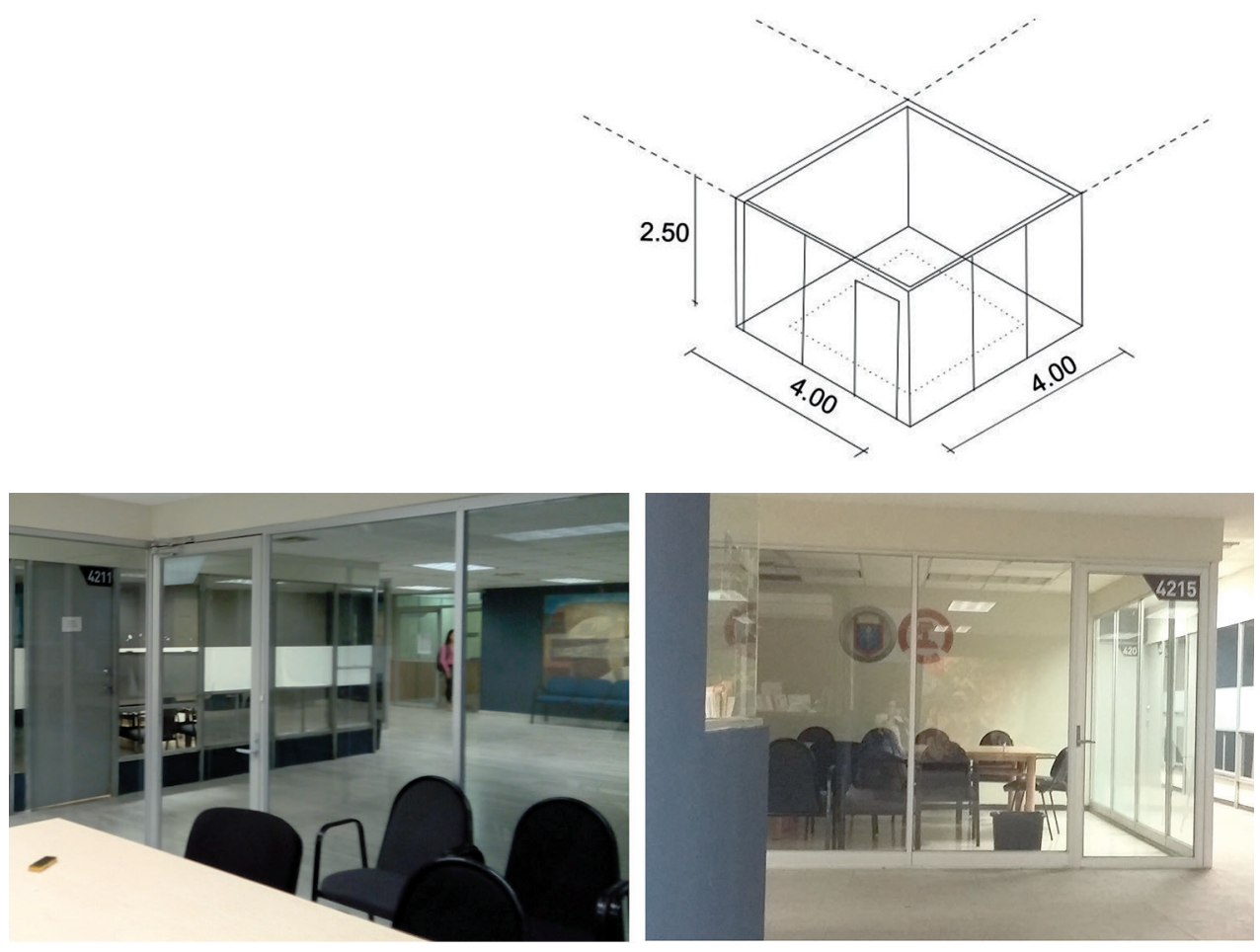

Figura 8. Isometría del aula A3 de la FARQ, UANL.

Figura 9. Fotos del aula A3 de la FARQ, UANL.

Fuente: autores.

Una vez descritas las tres aulas consideradas de alto potencial para inducir la creatividad en razón de sus características arquitectónicas, a continuación se presentan las tres aulas que fueron evaluadas como de bajo potencial creativo. 
Para el aula B1 se encuestaron 15 estudiantes de segundo semestre. Ubicada en el tercer nivel del edificio de laboratorios, mide $5 \mathrm{~m}$ ancho, $4 \mathrm{~m}$ de fondo y $2.50 \mathrm{~m}$ de altura al cielo de concreto armado de color blanco. Sus muros son de tableros de yeso, pintados de beige claro. Cuenta con luz artificial de lámparas de vapor de mercurio y buena iluminación natural, si bien solo en uno de sus lados hay ventanas de vidrio claro con cancelería de aluminio, las cuales pueden abrirse para obtener ventilación natural. Además, posee aire acondicionado.
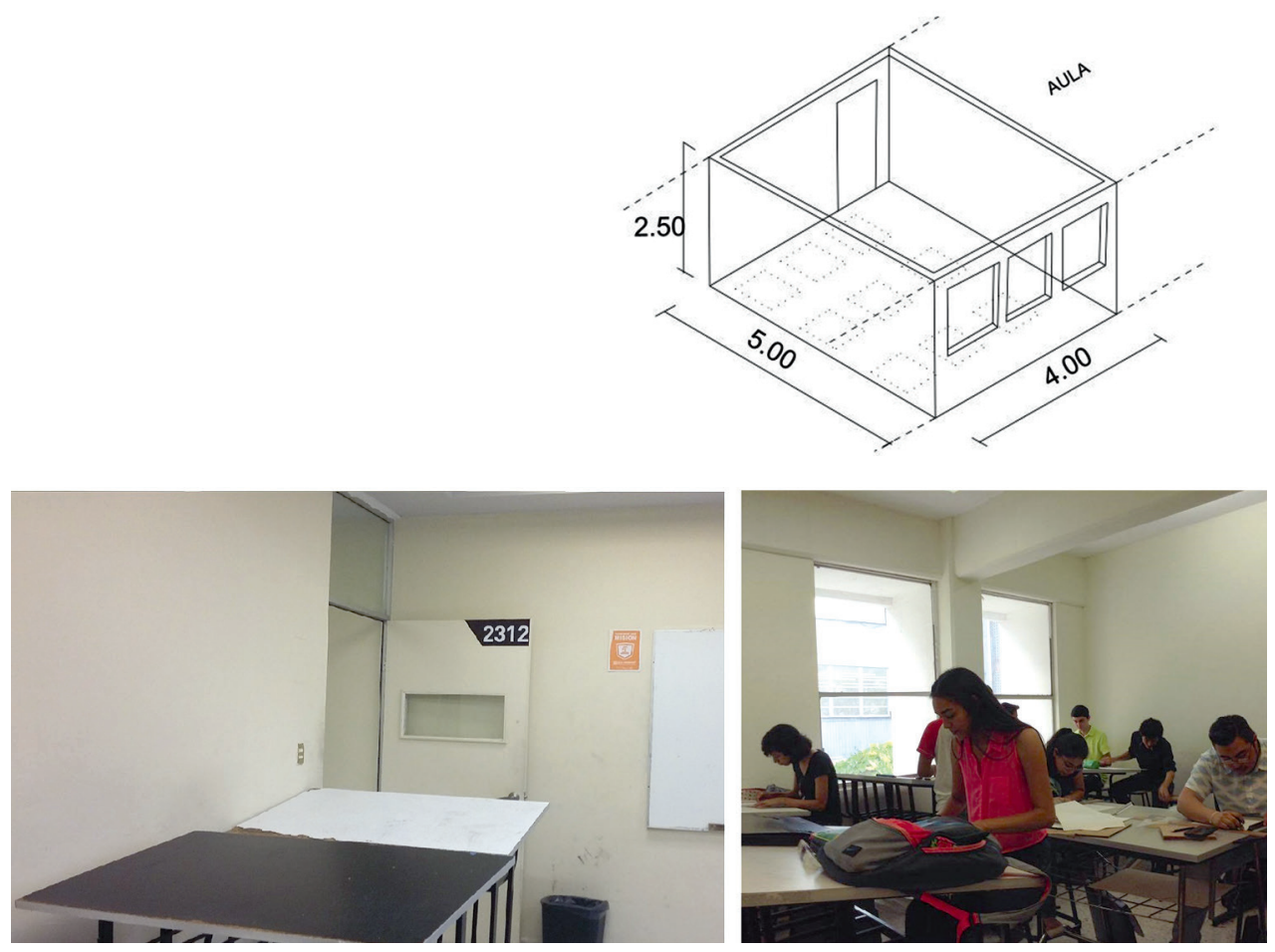

Figura 10. Isometría del aula B1 de la FARQ, UANL. Figura 11. Fotos del aula B1 de la FARQ, UANL. 
El aula B2, ubicada en el tercer nivel del edificio principal, es de las más grandes de toda la Facultad, con dimensiones de $11 \mathrm{~m}$ por $13 \mathrm{~m}$ y $2.70 \mathrm{~m}$ de altura al plafón de yeso de color blanco. Sus materiales interiores son tableros de yeso, pintados de gris claro en todos sus muros. Cuenta con ventanas únicamente en uno de los cuatro muros, sobre un antepecho de $1.30 \mathrm{~m}$. Tiene buena iluminación natural y artificial, con lámparas de vapor de mercurio distribuidas en todo el cielo. La ventilación se logra mediante aire acondicionado. Tal como comentan los 12 estudiantes de primer semestre que fueron encuestados, debido al gran tamaño se generan problemas de acústica, razón por la cual quienes se sitúan en los últimos asientos de la parte posterior no escuchan bien a los profesores.
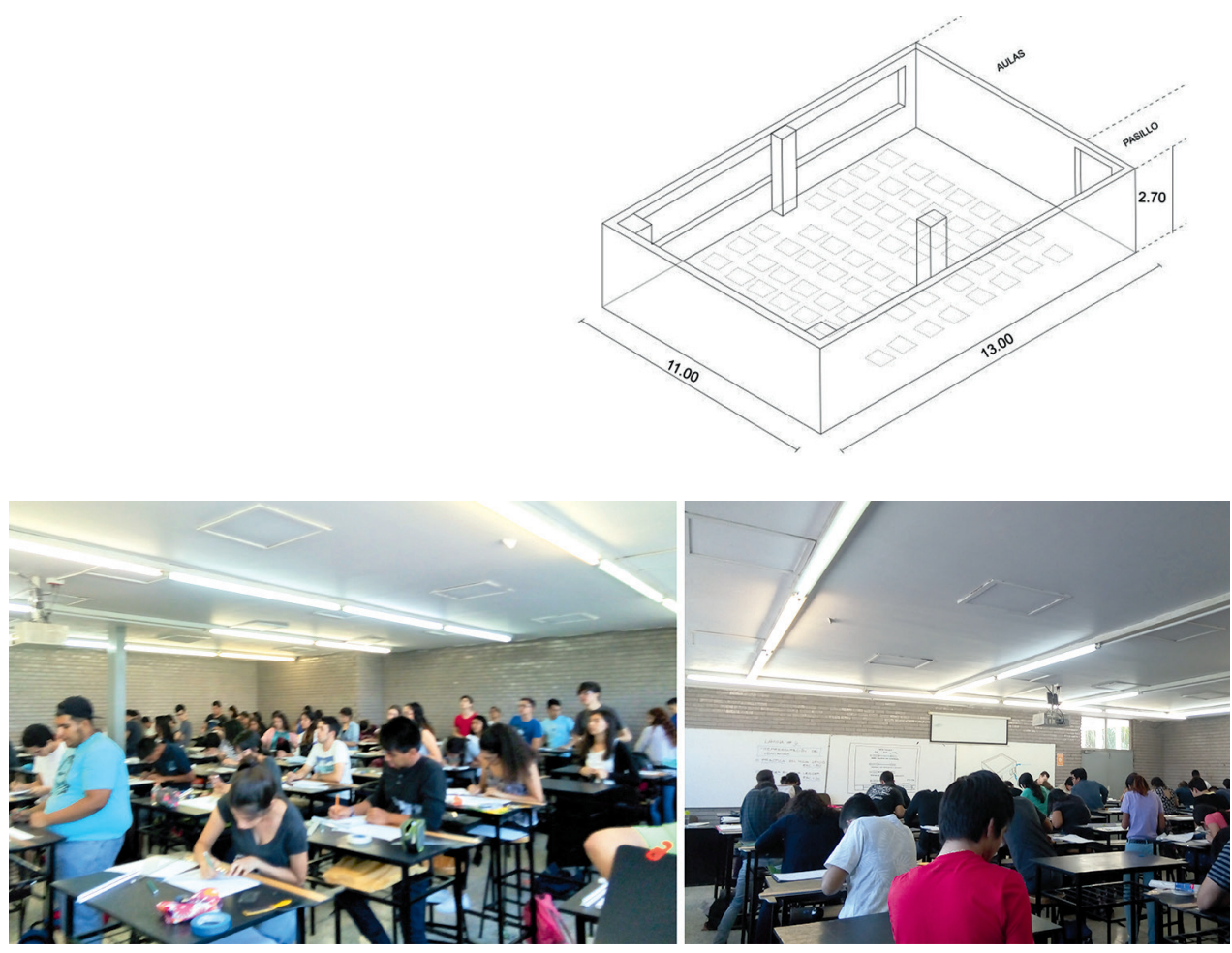

Figura 12. Isometría del aula B2 de la FARQ, UANL.

Figura 13. Fotos del aula B2 de la FARQ, UANL. 
Diez estudiantes de cuarto semestre respondieron la encuesta para el aula B3. Ubicada en la entrada del área de posgrado, mide $3 \mathrm{~m}$ de largo por $3.50 \mathrm{~m}$ de fondo y $2.50 \mathrm{~m}$ de altura al nivel del plafón modular de yeso de color blanco, el cual contiene lámparas de vapor de mercurio. Dos de sus muros son de vidrio reflectante de piso a techo, condición que impide ver hacia el exterior, donde está la recepción de posgrado. En el lado opuesto a estos se encuentra otro muro con ventanas de vidrio translúcido sobre un antepecho de $1 \mathrm{~m}$ de alto. Las ventanas pueden abrirse para permitir la ventilación natural y hay aire acondicionado.
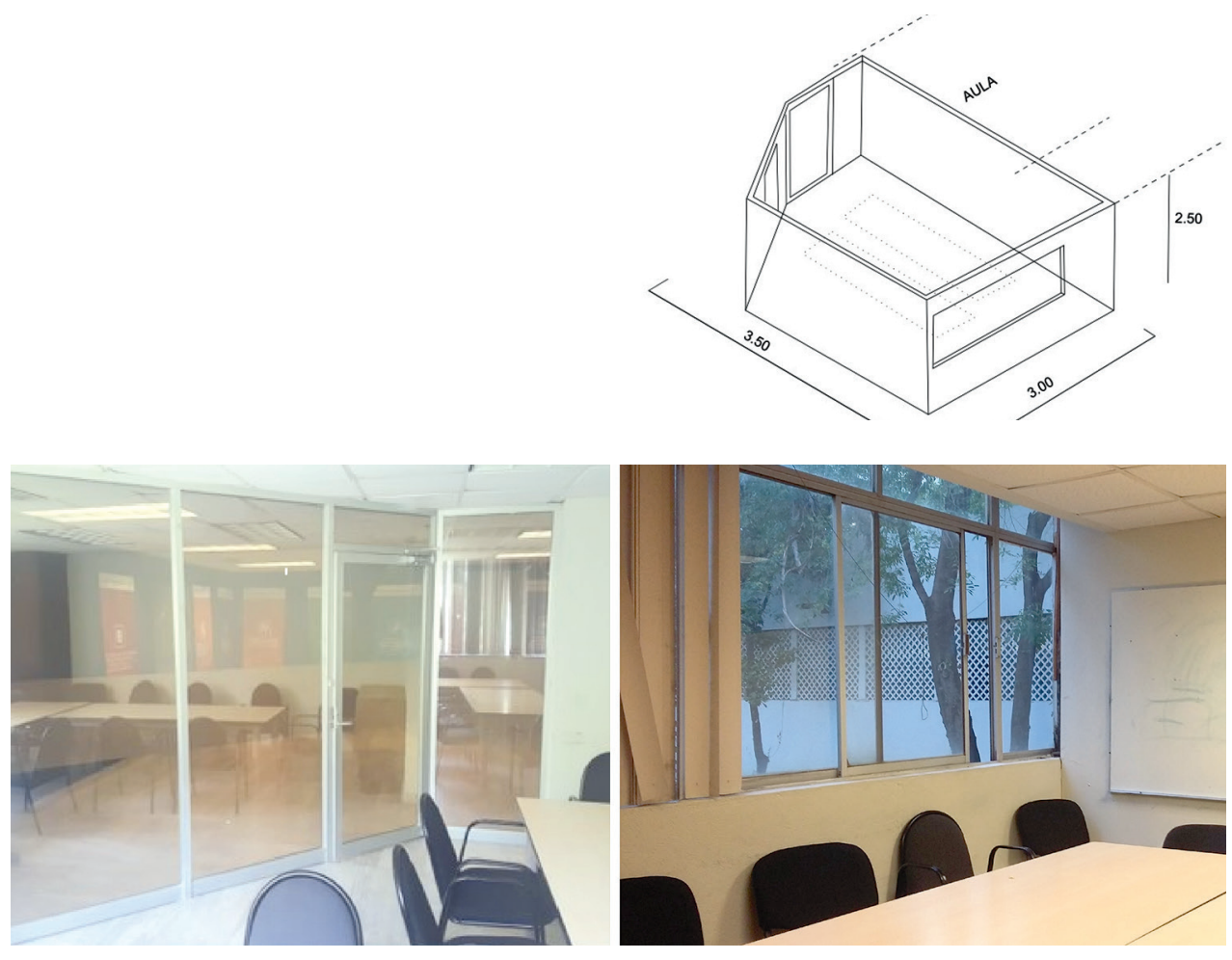

Figura 14. Isometría del aula B3 de la FARQ, UANL.

Figura 15. Fotos del aula B3 de la FARQ, UANL.

Fuente: autores.

Posteriormente, se efectuó el análisis estadístico de la información obtenida. En la tabla 2 se presentan datos para cada una de las preguntas formuladas. La altura obtuvo la media general más alta entre todas las aulas, pues el valor de 84.16 refleja que, en su mayoría, esta cualidad se percibe como adecuada. En contraste, el diseño creativo del espacio presentó la media más baja (49.59). 
Para verificar la confiabilidad de la encuesta se usó un análisis de consistencia interna Alfa de Cronbach. Los alfa registrados indican para todas las preguntas una confiabilidad satisfactoria, con un aceptable coeficiente de fiabilidad (0.944).

\begin{tabular}{|c|c|c|c|c|c|c|c|}
\hline \multirow{2}{*}{ PREGUNTA } & \multirow{2}{*}{$\begin{array}{l}\text { Media } \\
\text { Por }\end{array}$} & \multicolumn{3}{|c|}{$\begin{array}{l}\text { Aulas reportadas } \\
\text { como mejores }\end{array}$} & \multicolumn{3}{|c|}{$\begin{array}{l}\text { Aulas reportadas } \\
\text { como peores }\end{array}$} \\
\hline & & A1 & A2 & A3 & B1 & B2 & B3 \\
\hline $\begin{array}{l}\text { 1. ¿Qué tan buenas son las condiciones } \\
\text { en las que se encuentra esta aula? }\end{array}$ & 7,052 & 7,193 & 9,345 & 9,408 & 6,393 & 6,125 & 2,889 \\
\hline $\begin{array}{l}\text { 2. ¿Qué tan agradable es el color de esta } \\
\text { aula? }\end{array}$ & 5,415 & 52 & 5,054 & 75 & 6,643 & 4,125 & 30 \\
\hline $\begin{array}{l}\text { 3. ¿Qué tan adecuado es el espacio con } \\
\text { el que cuentas dentro del aula? }\end{array}$ & 7,182 & 7,493 & 8,482 & 8,308 & 6,571 & 8,258 & 30 \\
\hline $\begin{array}{l}\text { 4. ¿Qué tanto se te facilita trabajar } \\
\text { en esta aula? }\end{array}$ & 687 & 7,357 & 7,409 & 7,538 & 7,143 & 8,167 & 2,333 \\
\hline 5. ¿Qué tan espaciosa es esta aula? & 7,075 & 7,357 & 75 & 7,731 & 7,214 & 8,708 & 2,778 \\
\hline $\begin{array}{l}\text { 6. ¿Qué tanto se percibe el sonido del } \\
\text { exterior dentro de esta aula? }\end{array}$ & 6,437 & 60 & 4,845 & 8,808 & 6,357 & 725 & 4,678 \\
\hline $\begin{array}{l}\text { 7. ¿Qué tan adecuada es la altura } \\
\text { de esta aula? }\end{array}$ & 8,416 & 8,607 & 8,491 & 9,462 & 8,429 & 9,208 & 5,444 \\
\hline $\begin{array}{l}\text { 8. ¿Qué tan a gusto te sientes dentro } \\
\text { de esta aula? }\end{array}$ & 7,097 & 7,579 & 85 & 8,462 & 6,321 & 7,833 & 2,889 \\
\hline $\begin{array}{l}\text { 9. ¿Qué tan confortable es el mobiliario } \\
\text { en esta aula? }\end{array}$ & 6,388 & 6,179 & 83 & 9,115 & 575 & 5,208 & 30 \\
\hline $\begin{array}{l}\text { 10. ¿Qué tan apropiada es la acústica } \\
\text { dentro de esta aula? }\end{array}$ & 6,873 & 7,243 & 5,755 & 9,192 & 6,964 & 725 & 3,667 \\
\hline $\begin{array}{l}\text { 11. ¿Qué tan adecuada es la iluminación } \\
\text { artificial dentro de esta aula? }\end{array}$ & 7,714 & 8,336 & 9,327 & 9,269 & 6,964 & 7,983 & 3,333 \\
\hline $\begin{array}{l}\text { 12. ¿Qué tan adecuada es la ventilación } \\
\text { de esta aula? }\end{array}$ & 6,034 & 7,679 & 5,818 & 45 & 6,286 & 8,042 & 2,889 \\
\hline $\begin{array}{l}\text { 13. ¿Qué tanto se te facilita tener ideas } \\
\text { creativas dentro de esta aula? }\end{array}$ & 65,08 & 7,107 & 6,418 & 8,423 & 6,143 & 6,958 & 2,889 \\
\hline $\begin{array}{l}\text { "14. ¿Qué tan apropiada es la iluminación } \\
\text { natural dentro de esta aula?" }\end{array}$ & 6,471 & 80 & 9,191 & 1,654 & 6,893 & 7,442 & 5,778 \\
\hline $\begin{array}{l}\text { 15. ¿Qué tan adecuada consideras la } \\
\text { distribución del mobiliario en esta aula? }\end{array}$ & 7,175 & 7,979 & 8,182 & 9,123 & 6,607 & 7,417 & 2,444 \\
\hline $\begin{array}{l}\text { "16. ¿Qué tanto se te facilita comprender } \\
\text { la clase dentro de esta aula?" }\end{array}$ & 7,255 & 7,971 & 6,655 & 9,292 & 625 & 8,042 & 4,444 \\
\hline $\begin{array}{l}\text { 17. ¿Qué tan adecuada es la temperatura } \\
\text { de esta aula? }\end{array}$ & 65,96 & 7,107 & 8,736 & 9,462 & 5,357 & 6,158 & 156 \\
\hline
\end{tabular}




\begin{tabular}{|l|r|r|r|r|r|r|r|}
\hline $\begin{array}{l}\text { 18. ¿Qué tanto se te facilita concentrarte } \\
\text { en esta aula? }\end{array}$ & 6,767 & 6,857 & 7,818 & 8,192 & 5,893 & 7,917 & 3,111 \\
\hline $\begin{array}{l}\text { 19. ¿Qué tanto del entorno exterior } \\
\text { percibes desde esta aula? }\end{array}$ & 6,895 & 6,929 & 63 & 8,885 & 5,964 & 75 & 5,333 \\
\hline $\begin{array}{l}\text { 20. ¿Qué tan creativo te parece el } \\
\text { diseño o aspecto de esta aula? }\end{array}$ & 4,959 & 5,429 & 55 & 8,846 & 3,929 & 3,625 & 1,333 \\
\hline \begin{tabular}{l} 
Medias generales \\
\hline
\end{tabular} & 6,759 & 7,180 & 7,381 & 8,159 & 6,404 & 7,161 & 3,269 \\
\hline
\end{tabular}

Tabla 2. Medias de las evaluaciones de la encuesta. Fuente: autores. Nota: la media de todas las medias de cada ítem (segunda columna desde la izquierda) es de 67.59. Los valores mayores a esta cifra se resaltan con gris

La tabla 3 muestra las tres características mejor y peor evaluadas de cada aula. Se observa que la altura es una característica con alta evaluación en la mayoría de espacios, en tanto las más bajas son el diseño y el color.

\begin{tabular}{|c|c|c|c|c|}
\hline \multirow[t]{2}{*}{ AULA } & \multicolumn{2}{|c|}{ CARACTERÍSTICAS MÁS ALTAS } & \multicolumn{2}{|c|}{ CARÁCTERÍSTICAS MÁS BAJAS } \\
\hline & Altura & 86 & Color & 52 \\
\hline \multirow[t]{3}{*}{ A1 } & Iluminación artificial & 83 & Diseño creativo & 54 \\
\hline & Iluminación natural & 80 & Mobiliario confortable & 61 \\
\hline & Buenas condiciones & 93 & Color & 50 \\
\hline \multirow[t]{3}{*}{ A2 } & Iluminación artificial & 93 & Acústica & 57 \\
\hline & Iluminación natural & 91 & Mobiliario confortable & 83 \\
\hline & Altura & 95 & Iluminación Natural & 16 \\
\hline \multirow[t]{3}{*}{ A3 } & Temperatura & 95 & Ventilación & 45 \\
\hline & Buenas condiciones & 95 & Color & 75 \\
\hline & Altura & 84 & Diseño creativo & 39 \\
\hline \multirow[t]{3}{*}{ B1 } & Espaciosa & 72 & Temperatura & 53 \\
\hline & Iluminación artificial & 69 & Mobiliario confortable & 58 \\
\hline & Altura & 58 & Diseño creativo & 36 \\
\hline \multirow[t]{3}{*}{ B2 } & Espaciosa & 54 & Color & 41 \\
\hline & Ventilación & 53 & Mobiliario confortable & 52 \\
\hline & Iluminación natural & 58 & Diseño creativo & 13 \\
\hline \multirow[t]{2}{*}{ B3 } & Altura & 54 & Temperatura & 16 \\
\hline & Entorno exterior & 53 & Distribución de mobiliario & 24 \\
\hline
\end{tabular}

Tabla 3. Características mejor y peor evaluadas de cada aula. 
Con el fin de conocer cómo se relacionan entre sí las diferentes cualidades se realizó un análisis de correlación de Pearson, tanto de manera general como particular para cada aula. La tabla 4 presenta las correlaciones positivas más altas entre las cualidades de los espacios y la concepción de ideas creativas.

\begin{tabular}{|c|l|c|}
\hline AULA & \multicolumn{1}{|c}{ Cualidad / Actividad } & Correlación \\
\hline A1 & Temperatura & 0.623 \\
\hline A2 & Color & 0.681 \\
\hline A3 & Temperatura & 0.623 \\
\hline B1 & Facilidad para comprender la clase & 0.857 \\
\hline B2 & lluminación natural & 0.53 \\
\hline B3 & Color & 0.862 \\
\hline
\end{tabular}

Tabla 4. Correlaciones positivas más altas entre las cualidades de las aulas y las ideas creativas Fuente: autores.

Los resultados indican que la temperatura, el color y la iluminación natural pueden ser potenciadores del proceso creativo. De hecho, las dos primeras condiciones ambientales aparecen dos veces. En el caso del color, las aulas con la mayor correlación son la A2 y la B3, cuyos muros son beige y gris claro, esto es, sus pinturas son diferentes a las de los otros espacios, que son gris oscuro.

Además del análisis de correlaciones de Pearson para cada aula se aplicó otro general para obtener lar relaciones más significativas. La tabla 5 muestra en sus tres primeras columnas, de izquierda a derecha, las correlaciones más altas entre tener ideas creativas y las cualidades del espacio, mientras que las correlaciones más elevadas entre estas cualidades y el resto de aspectos aparecen en las dos columnas de la derecha. Puede verse que dos actividades y tres características tienen una correlación alta con la creatividad. Todas las correlaciones seleccionadas fueron significativas en el nivel 0.01 bilateral. 


\begin{tabular}{|c|c|c|c|c|}
\hline \multirow{24}{*}{$\begin{array}{l}\text { TENER IDEAS } \\
\text { CREATIVAS }\end{array}$} & \multirow{7}{*}{0.684} & \multirow{7}{*}{ Facilidad para trabajar } & 0.763 & Espacio adecuado \\
\hline & & & 0.676 & Distribución del mobiliario \\
\hline & & & 0.669 & Iluminación artificial \\
\hline & & & 0.661 & Altura \\
\hline & & & 0.627 & Ventilación \\
\hline & & & 0.62 & Temperatura \\
\hline & & & 0.601 & Acústica \\
\hline & \multirow{4}{*}{0.66} & \multirow{4}{*}{ Sentirse a gusto } & 0.806 & Facilidad para trabajar \\
\hline & & & 9.648 & Altura \\
\hline & & & 0.646 & Mobiliario confortable \\
\hline & & & 0.626 & Acústica \\
\hline & \multirow{6}{*}{0.648} & \multirow{6}{*}{ Altura } & 0.698 & Sentirse a gusto \\
\hline & & & 0.675 & Distribución del mobiliario \\
\hline & & & 0.665 & Iluminación artificial \\
\hline & & & 0.629 & Comprender la clase \\
\hline & & & 0.622 & Acústica \\
\hline & & & 0.600 & Temperatura \\
\hline & \multirow{4}{*}{0.643} & \multirow{4}{*}{$\begin{array}{l}\text { Distribución de } \\
\text { mobiliario }\end{array}$} & 0.775 & Iluminación artificial \\
\hline & & & 0.75 & Sentirse a gusto \\
\hline & & & 0.676 & Facilidad para trabajar \\
\hline & & & 0.675 & Altura \\
\hline & \multirow{3}{*}{0.637} & \multirow{3}{*}{ Acústica } & 0.626 & Sentirse a gusto \\
\hline & & & 0.622 & Altura \\
\hline & & & 0.601 & Facilidad para trabajar \\
\hline
\end{tabular}

Tabla 5. Correlaciones más altas entre tener ideas creativas y las cualidades del aula y entre estas con el resto de aspecto.

Fuente: autores.

\section{DISCUSIÓN}

De acuerdo con las correlaciones evidenciadas a partir del análisis particular y del general, aspectos como la altura, el sonido exterior, la temperatura, el color y la iluminación natural están ligados con el correcto funcionamiento del ambiente creativo. Los resultados estadísticos reflejan que la adecuada altura de las aulas se conecta con la capacidad para tener ideas creativas. Esto se encuentra en línea con los estudios de Lindwell (2011) y Vartanian (2013), referentes a cómo los espacios altos conducen a la abstracción y la creatividad. 
Según los resultados, en la percepción de la altura se considera el área de las aulas, pues aquellas en que esta cualidad fue evaluada de regular a buena poseen superficies entre 60 y $143 \mathrm{~m} 2$, en tanto la de $10 \mathrm{~m} 2$ fue la mejor calificada en este particular. Posiblemente, a menor planta se percibe mayor altura.

Respecto al color, las aulas analizadas poseen, en su mayoría, un homogéneo gris claro, pero son aquellas que no corresponden a este patrón, es decir, la B1, con sus muros beige claro, y la A3, dotada de dos muros contiguos de vidrio, las calificadas con los mayores puntajes en cuanto a color agradable. No obstante, se requiere mayor evidencia para señalar en qué medida esta cualidad resulta apropiada para los procesos de aprendizaje y las actividades creativas.

El sonido exterior, en la mayoría de aulas, fue calificado por debajo de la media, con 64.32. Esto indica que es poco percibido al interior de los espacios. A3 tuvo el valor más alto (88.08) de dicha percepción, lo cual podría deberse a que dos de sus muros son de vidrio, como también a su ubicación cercana a la entrada del departamento de posgrado, donde el flujo de estudiantes es constante.

Las seis aulas cuentan con sistema de ventilación artificial y sus temperaturas en el interior oscilan entre $23^{\circ} \mathrm{C}$ y $25^{\circ} \mathrm{C}$. La media más baja fue para B3, pues solo dispone de un ducto de ventilación que no funcionaba al momento de realizar la encuesta, justamente en temporada de calor y al medio día, con $32^{\circ} \mathrm{C}$. Los alumnos expresaron su incomodidad de trabajar en tales condiciones.

En cuanto a la luz natural, las aulas, en su mayoría, obtuvieron un puntaje por debajo de la media (65.08), con excepción de A2, cuya media fue más alta (91.91), en razón a sus grandes ventanas y su ubicación en el cuarto nivel con vista panorámica de la ciudad universitaria.

\section{CONCLUSIONES}

El presente estudio es un primer acercamiento al tema. Los datos obtenidos conducen a una reflexión sobre el papel de las cualidades arquitectónicas y ambientales de las escuelas de diseño en las actividades creativas. A su vez, posiblemente den lugar a investigaciones más profundas sobre la relación de la creatividad con el color, la iluminación, la temperatura, la acústica y la altura en las aulas. Tales cualidades pueden potenciar o bloquear la creatividad pero su identificación posibilita la realización de cambios que al generar lugares de trabajo más agradables para los estudiantes propicien la creatividad. 
REFERENCIAS BIBLIOGRÁFICAS

Arango-Díaz, L. (2016). Deslumbramiento en ambientes educativos con muro calado en fachada. AUS, (20), 62-69. Recuperado de http://mingaonline.uach.cl/scielo.php?script=sci_arttext\&pid=S0718-72622016000200010\&lng $=$ es\&nrm=iso

Csikszentmihalyi, M. (1998). Creatividad: el fluir y la psicología del descubrimiento y la invención. Barcelona: Paidós.

Hedge, A. (2004). Linking Environmental Conditions to Productivity. Presentation at the Eastern Ergonomics Conference and Exposition, New York, USA. Recuperado de https://studylib.net/doc/8683045/ ieq-and-productivity--is-there-a-link $\% 3 \mathrm{~F}$

Hernández, R., Fernández, C. \& Baptista, R. (2014). Metodología de la investigación. México D. F.: McGraw-Hill Education.

Kahn, L. (2015). Louis I. Kahn in conversation: Interviews with John W. Cook and Heinrich Klotz, 1969-70. New Haven: The Yale Center for British Art.

Kivi, R. (2017). How Does Color Affect Learning? Recuperado de http://classroom.synonym.com/color-affectlearning-4570805.html

Lindwell, W. (2011). Principios universales de diseño: 125 maneras de fomentar la facilidad de uso, influir en la percepción, incrementar el atractivo de los objetos, diseñar de forma más acertada y enseñar a través del diseño. Barcelona: Blume.

Mehta, R. (2012). Is Noise Always Bad? Exploring the Effects of Ambient Noise on Creative Cognition. JCR, 36(4), 784-799. Recuperado de http://www.jstor.org/stable/10.1086/665048

Rui, Z. (2009). Blue or Red? Exploring the Effect of Color on Cognitive Task Performances. Science Mag, University of British Columbia. Recuperado de http://science.sciencemag.org/content/323/5918/1226.full

Seppanen, O., Fisk, W. J. \& Lei, Q. H. (2005). Effect of temperature on task performance in office environment. Recuperado de https://www.researchgate.net/publication/237595590_Effect_of_temperature_on_task_performance_in_office_environment

Steidle, A. \& Werth, L. (2013). Freedom from constraints: Darkness and dim illumination promote creativity. Elsevie, 35, 67-80. Recuperado de http://www.sciencedirect.com/science/article/pii/S0272494413000261

Vartanian, O. (2013). Neuroscience of Creativity. MIT Press.

Vigovsky, E. (2011). Un ambiente propicio para lograr la tendencia creativa. Reflexión Académica en Diseño y Comunicación, 16, 13-16. Recuperado de https://fido.palermo.edu/servicios_dyc/publicacionesdc/archivos/270_libro.pdf 